\title{
Influence of the composition of different resin composites in the Knoop hardness and bond strength between tooth/restoration
}

\author{
Virgínia Bosquiroli ${ }^{1}$, William C Brandt ${ }^{2 *}$, Letícia C Boaro ${ }^{2}$, Isaías D Silva ${ }^{2}$ and Mário A Sinhoreti ${ }^{3}$
}

\author{
* Correspondence: \\ williamcbrandt@yahoo.com.br \\ ${ }^{2}$ School of Dentistry, University of \\ Santo Amaro (UNISA), Adress: Prof. \\ Eneas de Siqueira Neto, 340, São \\ Paulo, SP, Brazil \\ Full list of author information is \\ available at the end of the article
}

\begin{abstract}
The aim of this study was to evaluate the Knoop Hardness $(\mathrm{KH})$ and bond strength (BS) among different resin composites. Three composites (Z100- 3 M/ESPE; Filtek Supreme-3 M/ESPE, Filtek Silorane-3 M/ESPE) were tested. Thirty bovine incisors $(n=10)$ were used. Conical cavities were prepared in the buccal surface of each tooth with a diamond bur (3131 KG Sorensen, Barueri, SP, Brazil) with a high-speed water-cooled hand-piece in a standard cavity preparation appliance $(2.0 \times 2.0 \times 1.5 \mathrm{~mm})$, resulting in a C-Factor of 2.2. Two adhesive systems were used according to the manufacturer's instructions (Single Bond 2 and Silorane System Adhesive - 3 M ESPE). The restorations were made respectively with Z100, Supreme and Silorane. The composites were inserted in a single increment and light cured with a LED unit, Freelight (3 M ESPE) for $40 \mathrm{~s}$. After photo-activation the specimens were stored at $37^{\circ} \mathrm{C}$ in distilled water during $24 \mathrm{~h} . \mathrm{KH}$ (HMV-2, Shimadzu) were performed in the top and bottom at each specimen. After that, the push-out test was performed with a universal testing machine (Model 4411, Instron). In the KH test the data were submitted to a two way ANOVA and Tukey's test ( $a=5 \%)$. The mean of KHN and standard deviations were: Top - Z100 (74.1/9.0); Supreme (58.4/3.6); Silorane (42.8/6.2) and Bottom - Z100 (66.7/13.6); Supreme (61.2/3.6); Silorane (40.0/3.0). In the BS test the data were submitted to one way ANOVA and Tukey's test $(a=5 \%)$. The BS mean and standard deviations were: Z100 (12.6/5.2), Supreme (20.9/6.3), Silorane (29.7/9.0). Z100 had the highest KHN at the top and it was statistically different from the bottom. Supreme and Silorane had no differences between top and bottom. Silorane showed the highest BS mean differing statistically from the others. Differences in the composition of composite resins can to influence in the Knoop hardness and bond strength of restorations.
\end{abstract}

Keywords: Resin composite; Micro-hardness; Fillers; Organic matrix

\section{Background}

Light cured resin composites are commonly used in daily clinical practice to restore anterior and posterior teeth, because of their many advantages: esthetic, bonding to tooth structure, and mechanical properties. However, these materials undergo significant volumetric shrinkage when polymerized [1].

Insertion of these contracting composites into bonded preparations induces the development of mechanical stress inside the material [2]. The stress is transmitted via bonded interfaces to tooth structures. In light cured composites, the fast conversion 
induces fast increase in composite stiffness, causing high shrinkage stress at the interface. Such stress may disrupt the bond between the composite and the cavity walls or may even cause cohesive failure of the restorative material or the surrounding tooth tissue, in addition to postoperative sensitivity [3].

The rate of monomer conversion depends upon many factors as photo-initiator chemistry, filler morphology, pigment and irradiance $\left(\mathrm{mW} / \mathrm{cm}^{2}\right)$. But the irradiance applied to the composite is fundamental, because it is a factor that can be controlled by the professional through modulated photo-activation methods, differently of the factors mentioned previously. The higher the irradiance the faster the monomer conversion and the higher the stress generation. Photo-activation using low irradiance could reduce the stress, because it would allow flow during the earlier stages of polymerization and enable a certain degree of polymer chain relaxation before reaching the rubbery stage [3-5].

Modulation of the luminous energy has been shown to be efficient in decreasing the shrinkage stress of dental composite polymerization, but its clinical use is difficult, because it increases the clinical time and is dependent on the irradiance of the light curing unit, which the dentist does not usually know. Moreover, these methods can reduce the stress, but they do not reduce the final shrinkage of the material [3-6].

Therefore, with the objective of decreasing polymerization shrinkage, and consequently, the stress generated at the tooth/restoration interface, new monomers have been studied and introduced into the composition of dental composites. The monomers BisGMA, BisEMA, UDMA and TEGDMA, can be substituted by alternative monomers that have low polymerization shrinkage [7-9].

Recently, a silorane-based composite (Filtek P90), a synthesized monomer starting from oxirane and siloxane, was introduced on the market. Silorane-based composites differ from the methacrylate-based composites due to the polymerization process that occurs via a cationic ring-opening reaction, which decreases the volumetric contraction of the composite when compared with other methacrylate-based composites, in which the polymerization reaction is for addition [7].

When methacrylate monomers are replaced by silorane, not only can the polymerization shrinkage be reduced, but also the stress caused by it. Thus, many problems related to composite restorations, such as micro-leakage and marginal staining, secondary caries and postoperative sensitivity can be overcome [8-10].

Some clinical studies show that different materials for dental restoration can influence the longevity of the restoration increase the marginal integrity, marginal discoloration or surface texture [11] and thus reduce the need to replace the dental restoration [12].

Therefore, the aim of this study was to evaluate the Knoop hardness and bond strength between tooth/restoration of conventional methacrylate- and silorane-based composites. The bond strength was evaluated by the push out test, which is very useful for verifying the effect of polymerization shrinkage on composite restorations and its influence on bond strength.

The hypotheses tested were:

i) The silorane-based composites could produce higher values of bond strength between tooth/restoration that methacrylate-based composites;

iii) The methacrylate-based composites will obtain higher Knoop hardness values that silorane-based composites. 


\section{Methods}

Table 1 shows the materials used in this study.

A light emitting diode light source (LED) Freelight 2 (3 M/ESPE) was used to photoactivated the composites. The irradiance was calculated using a power meter and a digital caliper rule.

\section{Restorative procedures}

Thirty bovine incisors free from cracks or any other kind of structural defect were selected under 20X magnification. The teeth were disinfected in $0.5 \%$ chloramines-T solution for 15 days and stored for less than 1 month in $0.9 \%$ saline solution. The bovine incisors were prepared and restored according described in Brandt et al., 2008 [3]. The crowns were cut off at the cement-enamel junction using a diamond disk (KG Sorensen, Barueri, SP, Brazil). All buccal surfaces were ground and flattened under water cooling with a 400, 600 and 1200 grit $\mathrm{SiC}$ paper to standardize the thickness in dentin surface. A diamond bur was used to partially grind the lingual face of the crown and then received the same ground and flattened protocol that was done at the buccal surface.

Conical cavities were prepared in the buccal surface of each tooth with a diamond bur (\#3131KG Sorensen, Barueri, SP, Brazil) with a high-speed water-cooled hand-piece in a standard cavity preparation appliance. The cavity presented a conical form $2.0 \mathrm{~mm}$ in height, with a diameter of $2.0 \mathrm{~mm}$ at the top and $1.5 \mathrm{~mm}$ at the bottom, resulting in a C-Factor of 2.2. The diamond bur was replaced after every fifth preparation.

Preparations were etched using 35\% phosphoric acid (Scotchbond Etchant, 3 M-ESPE, St. Paul) and Single Bond 2 adhesive system (3 M-ESPE, St. Paul) was applied according to manufacturer's instructions and photo-activated for $10 \mathrm{~s}$. Ten restorations were made respectively with Z100- 3 M/ESPE; Filtek Supreme- 3 M/ESPE in A2 shade. The composite was placed in bulk mode (to increase the challenge caused by polymerization shrinkage of restorative material) and a mylar strip was placed over the cavity. Microscopy slice acrylic was used to force the composite to adapt to the preparation walls and to extrude the excess material. The slice was then removed and the light curing tip was positioned against the mylar, followed by photo-activation with a LED unit, Freelight (3 M/ESPE) for $40 \mathrm{~s}$. Finally, ten restorations were made using Silorane system adhesive according to manufacturer's instructions and photo-activated for $10 \mathrm{~s}$. The composite Filtek Silorane (3 M/ESPE) was applied with the same protocol as the made with the

Table 1 Description of the materials used according to the manufacturer - $3 \mathrm{M} / \mathrm{ESPE}$

\begin{tabular}{|c|c|c|c|}
\hline Resin composite & $\begin{array}{c}\text { Adhesive system } \\
\text { used }\end{array}$ & Organic matrix & $\begin{array}{c}\text { Filler content - Wt } \% \text { - } \\
\text { Average size }\end{array}$ \\
\hline $\mathrm{Z100}$ & Adper Single Bond2 & Bis-GMA* and TEGDMA* & $\begin{array}{c}\text { Zirconia and silica - 84.5\% - } \\
0.6 \mu \mathrm{m}\end{array}$ \\
\hline Filtek Supreme & Adper Single Bond2 & $\begin{array}{c}\text { Bis-GMA, Bis-EMA (6)* }{ }^{*}, \text { UDMA }^{*} \text { and } \\
\text { TEGDMA }\end{array}$ & $\begin{array}{c}\text { Zirconia and silica - } 78.5 \%- \\
\text { Nanofillers (5-20 } \mathrm{nm}) \text { and } \\
\text { Clusters (0.6 to } 1.4 \mu \mathrm{m})\end{array}$ \\
\hline Filtek Silorane & $\begin{array}{l}\text { Silorane System } \\
\text { Adhesive }\end{array}$ & $\begin{array}{c}\text { Silorane (3,4-epoxy cyclohexylethyl } \\
\text { siloxane, bis-3,4- epoxy cyclohexylethyl } \\
\text { phenylmethyl silane) }\end{array}$ & $\begin{array}{l}\text { Quartz and yttrium - 76\%- } \\
0.47 \mu \mathrm{m}\end{array}$ \\
\hline
\end{tabular}

*Bis-GMA: bisphenol glycidyl methacrylate, TEGDMA: triethyleneglycol dimethacrylate, UDMA: urethane dimethacrylate, Bis-EMA: bisphenol ethoxylate dimethacrylate. Bis-EMA (6) is analogous to Bis-GMA; however, the former has six ethylene oxide units substituting the two hydroxyl groups between the aromatic backbone and the insaturates. 
other resins. After light curing, the specimens were stored at $37^{\circ} \mathrm{C}$ in distilled water during $24 \mathrm{~h}$.

Additionally, representative specimens were prepared for evaluating the thickness of the adhesive layer (Figure 1). Two additional bovine incisors of each group were restored as previously described and were considered. Images were digitized using a 60x magnifying stereomicroscope (model SZ61, Olympus Inc., Tokyo, Japan) equipped with a CCD camera (Q-Color 3, Olympus).

\section{Knoop hardness test}

After $24 \mathrm{~h}$ the top and bottom sample surfaces were polished under water with a $\mathrm{SiC}$ 1200 sandpaper to obtain flattened surface. The indentations and micro-hardness measurements (KHN) (HMV-2, Shimadzu, Tokyo, Japan) were performed, five times at each specimen surface (top and bottom) with a 50 gf load during $15 \mathrm{~s}$ and the mean hardness value was obtained for each surface by the average of the five indentations.

To avoid samples dehydration and consequences on teeth/composite bond values, the KHN readings were made alternately at each surface of the samples in order to maintain the hydration. First the top surface was made and the sample was putted back to the water recipe while the reading was performed at another sample. Later the bottom reading at the first sample was made and successively.

\section{Bond strength test}

Bond strength was evaluated using a push-out test. The sample was positioned on top of a metallic device that had an aperture that allowed the smaller diameter of the restoration to be in contact with a cylindrical device, connected to the load cell of a universal testing machine (Instron, model 4411, Buckinghamshire, England). This cylindrical device applied a compressive force on the smaller diameter surface of the restoration, until rupture of the tooth-composite bond was achieved. The push-out test was carried out at a cross-head speed of $0.5 \mathrm{~mm} / \mathrm{min}$. Values in MPa were obtained by dividing maximum load by the bonded surface area of each specimen. The cylindrical device was positioned, thus to touch only the middle of the restoration and by the way it will not overestimate the results by using the lateral walls of the cavity.

\section{Statistical analysis}

Kolmogorov-smirnov test was made to evaluated normality and homogeneity and after the KHN data were analyzed using two-way ANOVA (composite and surface) and
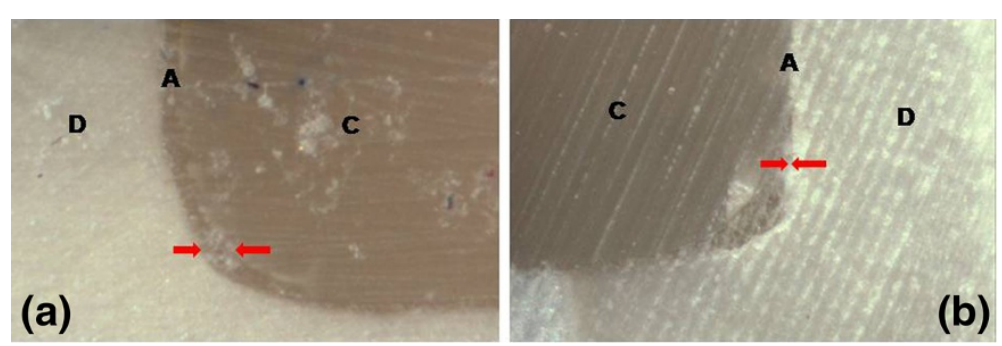

Figure 1 Stereoscope microscope images in the restorations of Silorane (a) and Z100 (b) showing the difference in thickness of the adhesive layer. The letters mean D-dentin, A-adhesive and C-composite. 
Tukey's test as post hoc test. BS data were analyzed using one-way ANOVA (composite) and Tukey's test. The significance level used was $\alpha=0.05$ for all analyses.

\section{Results}

According to statistical analysis, there is interaction between the factors analyzed (composite and surface) for the KHN data $(\mathrm{p}=0.041)$.

Results for KHN are shown in Table 2. For the Z100 composite, the Knoop hardness in the top was higher and statistically different from the bottom $(\mathrm{p}<0.001)$. For Supreme and Silorane, there was no difference in the values of KHN between the top and bottom $(\mathrm{p}=0.869)$. At the top region, $\mathrm{Z} 100$ composite shows the highest KHN means and statistically different from the others following for Supreme and the lowest value for Silorane. At the bottom region, Silorane also shows the lowest mean of KHN and Supreme and Z100 composite did not differ one from the other.

According to statistical analysis, there is differences between the BS data $(\mathrm{p}=0.006)$.

Results for BS are shown in Table 3. Silorane shows the highest BS values $(\mathrm{p}<0.001)$ and Z100 showed the lowest BS values $(\mathrm{p}<0.001)$. Supreme showed intermediary values with statistical difference.

Stereomicroscope images showed that the thickness of the adhesive layer of the Silorane system adhesive is thicker than Single Bond 2.

\section{Discussion}

Despite the frequent increase of restorations made of composite resin, there is still no material considered ideal. Therefore, changes in the composition of the composites are often performed in an attempt to obtain this ideal material [13]. Among the most desirable qualities in composites, we can mention the increase in mechanical properties, maintaining aesthetics and low or no polymerization shrinkage.

The polymerization shrinkage of dental composites is still the main cause of flaws in restorations. The shrinkage of the material can cause post-operative sensitivity and/or debonding, and consequently, marginal staining, microleakage and secondary caries [14]. Then, several researchers have endeavored to reduce the shrinkage stress with the objective of reducing the problems caused by polymerization shrinkage, which is inherent to the material [2-6].

In this study, three different restorative materials (Z100, Supreme and Silorane) were used because they have differences in their composition. These differences in composition occur in the organic matrix and in the filler type of each composite. Z100 is a methacrylate-microhybrid with Bis-GMA/TEGDMA monomers, Supreme is a methacrylate-nanofilled with UDMA/Bis-EMA monomers and Silorane is a Siloranemicrohybrid with silorane monomers.

Table 2 Hardness Knoop (KHN $-\mathrm{Kgf} / \mathrm{mm}^{2}$ ) means values and standard deviations of the top and bottom in the three resin composites

\begin{tabular}{llll}
\hline Composite region & Z100 & Supreme & Silorane \\
\hline Top & $74.1(9.0) \mathrm{a}, \mathrm{A}$ & $58.4(3.6) \mathrm{a}, \mathrm{B}$ & $42.8(6.2) \mathrm{a}, \mathrm{C}$ \\
Bottom & $66.7(13.6) \mathrm{b}, \mathrm{A}$ & $61.2(3.4) \mathrm{a}, \mathrm{A}$ & $40.0(3.0) \mathrm{a}, \mathrm{B}$ \\
\hline
\end{tabular}

Means followed by distinct lowercase letters in collum and distinct uppercase letters in line are statistically different in $5 \%$ level signifficance by Tukey test. 
Table 3 Bond strenght (BS) mean values (MPa) and standard deviations of the three composites

\begin{tabular}{lll}
\hline Resin composite & Mean (sd) & Tukey (5\%) \\
\hline Silorane & $29.7(9.0)$ & $\mathrm{A}$ \\
Supreme & $20.9(6.3)$ & $\mathrm{B}$ \\
Z100 & $12.6(5.2)$ & $\mathrm{C}$ \\
\hline
\end{tabular}

Means followed by distinct uppercase letters in collum are statistically different in $5 \%$ level signifficance by Tukey test.

Because of these differences in the composition of the composites evaluated, they showed some different results with each other in the evaluated tests. The siloranebased composite Silorane showed higher bond strength values than methacrylate-based composites Supreme and Z100. Consequently, the first hypothesis was accepted.

Silorane composite has a silorane network, which is generated by the cationic ring opening polymerization of the cycloaliphatic oxirane moieties, which stand for their low shrinkage and low polymerization stress. The low polymerization shrinkage and shrinkage stress can lead to an increase in the bond strength $[7,10,15]$.

Different adhesive systems were used. Silorane has an own adhesive system, because possesses a different composition to the methacrylate-based composites as Supreme and Z100. Silorane system adhesive is a self-etch adhesive differently of Single Bond 2 used with Supreme and Z100 that is an etch-and-rinse adhesive. The use of different adhesive systems might have contributed to the differences found in the bond strength values. During the restorative procedure was possible to visualize the differences in the thickness of the adhesive layer. According images made in stereoscope (Figure 1), it was found that Silorane system adhesive obtained thicker layer than Single Bond 2. This thicker layer of adhesive may have contributed to the higher values of BS for the composite Silorane because it could function as an elastic layer which reduces the stress of polymerization. However, further studies verifying the thickness of adhesive on BS should be performed [10].

The higher BS seen in Supreme when compared with Z100 can be explained by the presence of UDMA and BisEMA (6), replacing part of TEGDMA in Z100. The shorter molecules of TEGDMA and the larger number of reacting aliphatic bonds in Z100 suggested that the Z100 matrix may shrink more than that of Supreme, and consequently produce lower values of BS [16].

The Knoop hardness showed that Silorane presented lower Knoop hardness means than Z100 and Supreme, which could suggest reduced mechanical properties. Thus, the second hypothesis was accepted.

The hardness of composite resins is particularly dependent on the filler type and content and it correlates with mechanical properties, such as abrasion resistance or polishability. The Silorane composite exhibited the lowest KHN, which is similar to previous studies $[17,18]$. When analyzed the KHN in the top, Z100 showed higher KHN values than Supreme. This is possible due to the greater amount of filler particles present in the composite Z100 (84.5 wt\%) in comparison to Supreme (78.5 wt\%). The KHN in bottom was lower than in top in the composite Z100, while in other composites, such as for example Supreme did not happen. Despite the lower number of reacting aliphatic groups in the Supreme (some of the monomers TEGDMA of Z100 were replaced by UDMA and BisEMA), it still seems that a more efficient reaction occurred 
in Supreme. The more efficient reaction most likely related to the fewer aliphatic groups which needed to be consumed before a high conversion value was reached. Another possibility could be that there were different photo-initiators used in these two composites (something that could also have affected the reaction). Then in deep regions, the polymerization reaction of Z100 can be impaired, thus showing reduced properties.

\section{Conclusion}

Differences in the composition of composite resins can to influence in the Knoop hardness and bond strength of restorations.

The composite resin Z100 showed the higher KHN values, but the lower BS values; while the composite resin Silorane showed the lower KHN values, but the higher BS values.

Competing interests

The authors declare that they have no competing interests.

\section{Authors' contributions}

BV carried out the hardness Knoop, the bond strength, participated in the sequence alignment and drafted the manuscript. BWC chose the materials and methodology, participated in the sequence alignment and drafted the manuscript. BLC performed the Stereoscope microscope images and participated in the sequence alignment and drafted the manuscript. SID participated in the sequence alignment and drafted the manuscript. SMAC participated in the sequence alignment and drafted the manuscript. All authors read and approved the final manuscript.

\section{Author details}

${ }^{1}$ Operative Dentistry and Prosthodontics Area, Dental School, Westerm Paraná State University, Cascavel - PR, Brazil.

${ }^{2}$ School of Dentistry, University of Santo Amaro (UNISA), Adress: Prof. Eneas de Siqueira Neto, 340, São Paulo, SP, Brazil. ${ }^{3}$ Department of Restorative Dentistry, Piracicaba Dental School, University of Campinas (UNICAMP), Adress: Av. Limeira, 901, Piracicaba, SP, Brazil.

Received: 14 November 2013 Accepted: 4 December 2013

Published: 4 August 2014

\section{References}

1. Ferracane $J \mathrm{~L}$ (2011) Resin composite-state of the art. Dent Mater 27(1):29-38

2. Davidson $\mathrm{CL}$, de Gee AJ (1984) Relaxation of polymerization contraction stresses by flow in dental composites. J Dent Res 63:146-148

3. Brandt WC, de Moraes RR, Correr-Sobrinho L, Sinhoreti MA, Consani S (2008) Effect of different photo-activation methods on push out force, hardness and cross-link density of resin composite restorations. Dent Mater 24:846-850

4. Segreto D, Brandt WC, Correr-Sobrinho L, Sinhoreti MA, Consani S (2008) Influence of irradiance on the push-out bond strength of composite restorations photoactivated by LED. J Contemp Dent Pract 9:89-96

5. Cunha LG, Alonso RC, Pfeifer CS, Correr-Sobrinho L, Ferracane JL, Sinhoreti MA (2007) Modulated photoactivation methods: Influence on contraction stress, degree of conversion and push-out bond strength of composite restoratives. J Dent 35(4):318-24

6. Alonso RC, Cunha LG, Correr GM, Cunha Brandt W, Correr-Sobrinho L, Sinhoreti MA (2006) Relationship between bond strength and marginal and internal adaptation of composite restorations photocured by different methods. Acta Odontol Scand 64:306-313

7. Weinmann W, Thalacker C, Guggenberger R (2005) Siloranes in dental composites. Dent Mater 21:68-74

8. Palin WM, Fleming GJ, Nathwani H, Burke FJ, Randall RC (2005) In vitro cuspal deflection and microleakage of maxillary premolars restored with novel low-shrink dental composites. Dent Mater 21:324-335

9. Eick JD, Kotha SP, Chappelow CC, Kilway KV, Giese GJ, Glaros AG, Pinzino CS (2007) Properties of silorane-based dental resins and composites containing a stress-reducing monomer. Dent Mater 23:1011-1017

10. Boaro LC, Gonçalves F, Guimarães TC, Ferracane JL, Pfeifer CS, Braga RR (2013) Sorption, solubility, shrinkage and mechanical properties of "low-shrinkage" commercial resin composites. Dent Mater 29(4):398-404

11. Gonçalves FS, Leal CD, Bueno AC, Freitas AB, Moreira NA, Magalhães CS (2013) A double-blind randomized clinical trial of a silorane-based resin composite in class 2 restorations: 18-month follow-up. Am J Dent 26(2):93-8

12. Pallesen U, van Dijken JW, Halken J, Hallonsten AL, Höigaard R (2013) A prospective 8-year follow-up of posterior resin composite restorations in permanent teeth of children and adolescents in Public Dental Health Service: reasons for replacement. Clin Oral Investig, in press 
13. Ferracane JL (2013) Resin-based composite performance: are there some things we can't predict? Dent Mater 29(1):51-8

14. Tantbirojn D, Versluis A, Pintado MR, DeLong R, Douglas WH (2004) Tooth deformation patterns in molars after composite restoration. Dent Mater 20(6):535-42

15. Brandt WC, Lacerda RF, Souza-Junior EJ, Sinhoreti MA (2013) Effect of photoactivation mode on the hardness and bond strength of methacrylate- and Silorane monomer-based composites. J Adhes Dent 15(1):33-9

16. Emami N, Söderholm KJ (2003) How light irradiance and curing time affect monomer conversion in light-cured resin composites. Eur J Oral Sci 111(6):536-42

17. Porto IC, de Aguiar FH, Brandt WC, Liporoni PC (2013) Mechanical and physical properties of silorane and methacrylate-based composites. J Dent 41(8):732-9

18. Leprince J, Palin WM, Mullier T, Devaux J, Vreven J, Leloup G (2010) Investigating filler morphology and mechanical properties of new low-shrinkage resin composite types. J Oral Rehabil 37:364-76

doi:10.1186/2196-4351-2-14

Cite this article as: Bosquiroli et al.: Influence of the composition of different resin composites in the Knoop hardness and bond strength between tooth/restoration. Applied Adhesion Science 2014 2:14.

Submit your manuscript to a SpringerOpen ${ }^{\odot}$ journal and benefit from:

- Convenient online submission

- Rigorous peer review

- Immediate publication on acceptance

- Open access: articles freely available online

- High visibility within the field

- Retaining the copyright to your article 